\title{
Ordering of hard spheres inside hard cylindrical pores
}

\author{
F. J. Durán-Olivencia and M. C. Gordillo \\ Departamento de Sistemas Físicos, Químicos y Naturales, Facultad de Ciencias Experimentales, Universidad Pablo de Olavide, \\ Carretera de Utrera, $\mathrm{km} \mathrm{1,} 41013$ Sevilla, Spain
}

(Received 13 February 2009; published 16 June 2009)

\begin{abstract}
Isothermal-isobaric simulations on the ordering behavior of hard spheres upon confinement are presented. The radii of the confining cylinders go from 1.1 to 2 in units of the diameters of the hard spheres adsorbed. In all the range of pressures considered the spheres were located in concentric layers, as many as the radius of the hard cylinder would permit. When the pressure increases, the hard spheres go from being loosely arranged to the formation of ordered structures. This change is marked in all cases by a distinct break in the density of spheres in a narrow pressure range. When the tube radius is smaller than 1.5, the high-pressure ordering is determined by the number of coplanar spheres you can have within a circle of radius equal to that of the confining tube. For wider tubes, the change upon compression is determined by the formation of defected two-dimensional triangular lattices wrapped to fit inside the particular cylinder we are considering.
\end{abstract}

DOI: 10.1103/PhysRevE.79.061111

PACS number(s): 05.20.Jj, 05.70.Fh, 61.20.Ja

\section{INTRODUCTION}

The behavior of simple fluids under confinement could be rather complex (see, for instance, Ref. [1] and references therein). This means that if we want to have a reasonable picture of it, we should take into account both the pure confining effects and the interaction of the constrained species with the walls of the confining media. For instance, it is well known that when the adsorbate-adsorbent interaction is greater that the adsorbate-adsorbate one, the freezing point of the confined species increases with respect to its bulk counterpart. The opposite happens when the relative order of the strength reverses [1-6]. When the confining walls are made of the same atoms or molecules than the adsorbate, the freezing point decreases with the size of the pore. This is common to both cylindrical and slit pores.

Another topic of study is the ordering of the adsorbed species inside the tube. Obviously, this should depend on the size of the pore and on the wall-adsorbate interaction. In cylindrical pores, the majority of the theoretical studies have been made on tubes of radius equal to or greater than $2 \sigma$, with $\sigma$ as the diameter of the adsorbed species [1,2,4-6], even though there are some simulations on or including narrower pores [7-12]. Among them, Refs. [7-9,11] are on the subject of hard spheres inside hard pores. Apart from its theoretical interest, these last results could be used to understand the recent experimental findings about colloids inside very narrow rectilinear pores $[13,14]$ and, to a lesser extent, the confining effects of other substances inside narrow tubes [15-17].

The aim of this work is to study the effect of confinement in the structure of hard spheres located inside hard tubes of radii in the range between 1.1 and $2 \sigma$ in a given range of reduced pressures. Here, and in the remainder of the paper, $\sigma$ will be the diameter of the hard spheres confined in the tubes, with all the lengths given in units of $\sigma$. Our goal is not to have the absolute maximum package ratio as in Ref. [8] but to consider the average equilibrium configurations of a set of hard spheres when the pressure increases, taking into account the influence of the entropy in the configurations obtained. The plan of this paper is as follows: in Sec. II we will describe the model and simulation details, while Sec. III will be devoted to describe the results obtained. Finally, we will state our conclusions in Sec. IV.

\section{MODEL AND SIMULATION DETAILS}

Our primary goal was to study the variation in the ordering of hard spheres located inside perfectly rigid hard tubes under compression in the $z$ direction (the main axis of the confining tubes). To do so, we performed isothermal-isobaric (NPT) Monte Carlo simulations in the reduced pressures range $P^{*}=0-20 . P^{*}$ was taken as the simplified form for $\beta P \sigma^{3}$, and it will be used in that way in the remainder of the paper. $\beta=1 / k_{B} T$, where $k_{B}$ is the Boltzmann constant and $T$ is the temperature. The radii of the confining tubes were $R$ $=1.2,1.3,1.4,1.5,1.7$, and $2 \sigma$. This means that the maximum distance of any sphere to the center of the tube should be lower or equal to $R-0.5 \sigma$. In all cases the number of particles was $N=800$, number that we checked to be sure that was enough to avoid size effects. To do so, we repeated some of the simulations in the region immediately below and above the density breakings (see below), keeping constant all the other variables but changing the number of spheres $(N$ $=1600,3200)$. We found no appreciable change in the variables tested, with the results similar to the ones for $N=800$ within a single standard deviation. All the data were obtained by averaging over $3 \times 10^{6}$ simulation steps. The number of discarded simulation steps varied from tube to tube but in all cases were at least $3 \times 10^{6}$, and enough to assure that in the $3 \times 10^{6}$ ones taken to calculate the results presented here, the volume of simulation cell had no particular bias. Each Monte Carlo step comprised an attempt to change the volume of the simulation cell, and $2 N$ trials to move a hard sphere chosen at random. The maximum movement distance was fixed to ensure a percentage of success around $40 \%$. The same control was applied to the change in the volume of the cell containing the spheres, i.e., a success ratio between $30 \%$ and $40 \%$. Since the cylinders were considered to be perfectly 
rigid, the change in the volume translated into a change in the length of the simulation cell.

We were interested in characterizing the change in the detailed distribution of the spheres within the cylinder upon increasing the axial pressure. To do so, we checked the possible formation of helical structures. A perfect helix is defined by the following expressions [8]:

$$
\begin{gathered}
d=R-0.5 \sigma, \\
z_{n}-z_{n-1}=a, \\
\Phi_{n}=\theta,
\end{gathered}
$$

where $d$ is the distance from the center of the tube to the position of the particle, $z_{n}$ is the position in the $z$ axis of the particular sphere considered $(n)$, with $z_{n}-z_{n-1}$ as the difference in the locations of two consecutive spheres in the same helix, and $\Phi_{n}$ is the angle between the two the centers of these two spheres and the center of the tube. If the helices are not perfect, $d, z_{n}-z_{n-1}$, and $\Phi_{n}$ should have some dispersion around a single maximum.

If the tube is wide enough, we can use an alternative description to that of the helices; any of the concentric layers we can see (if more than one) can be seen as the result of the wrapping up of a two-dimensional triangular lattice inside the tube. That structure will have more or less defects depending on the distance of this cylindrical shell to the center of the tube and on the external pressure. To quantify the quality of this approximation, we calculated the hexatic order parameter as $\left|\left\langle\Phi_{6}\right\rangle\right|$, where

$$
\Phi_{6}=\frac{1}{N} \sum_{i}^{N} \frac{1}{N_{i c}} \sum_{j, k}^{N_{i b}} \exp \left(i 6 \theta_{j k}\right) .
$$

The average over the parameter was made over the respective simulation runs. In principle, $\Phi_{6}$ would depend on the cutoff distance to define the nearest neighbors of each atom $i$. To avoid that, different cutoff distances were tried to assure that a particular election did not change the results. $\theta_{j k}$ is the angle defined by the atoms $j, i$, and $k$, with $j$ and $k$ as two of the nearest neighbors of atom $i . N_{i b}$ is the number of the closest neighbors of atom $i$, and $N_{i c}$ is the total number of the corresponding angles for the same atom. $\left|\left\langle\Phi_{6}\right\rangle\right|$ was calculated with the actual positions of the hard spheres in the corresponding layer without any projection in a twodimensional plane.

\section{RESULTS}

\section{A. Narrow cylinders $(R<1.35 \sigma)$}

In this subsection we will characterize the ordering upon compression of sets of hard spheres confined in tubes narrow enough to allow at most one layer of those spheres inside, starting by Fig. 1. There, we show the equation of state (EOS) corresponding to a tube of radius $1.2 \sigma$. There, the $y$ axis represents the applied reduced pressure $\left(\beta P \sigma^{3}\right)$ and in the $x$ one we displayed the reduced density as the number of spheres divided by the volume of the simulation cell. This

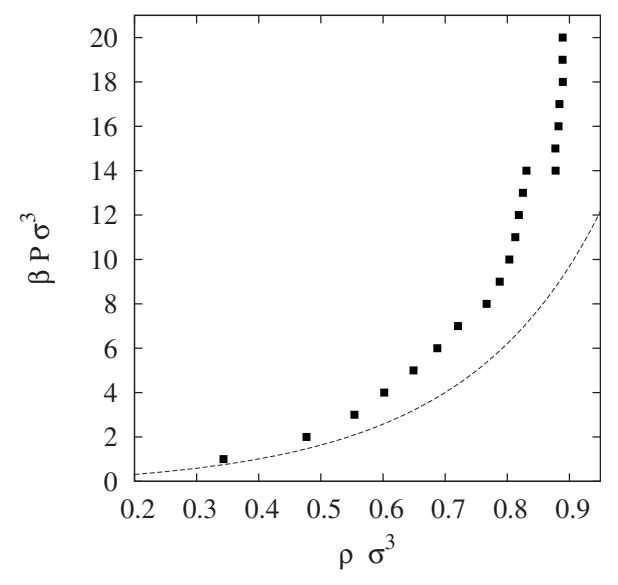

FIG. 1. Equation of state for hard spheres confined inside a hard cylinder of radius $1.2 \sigma$. Dashed line indicates the same quantity for a three-dimensional bulk system in the same density range. $\beta P \sigma^{3}$ means reduced pressure $\left(\beta=1 / k_{B} T ; k_{B}\right.$ : Boltzmann constant; $T$ : temperature), while $\sigma$ is the diameter of the hard spheres. $\rho$ is the number density, i.e., the number of spheres divided by the cylinder volume $\left(1.44 \pi \sigma^{3} L\right.$, where $L$ is the average length of the simulation cell in units of $\sigma$ ).

volume is expressed in units of the cube of the length unit, $\sigma$. The error bars are of the size of the symbols and not shown for simplicity. The form of the curve is similar to the EOS of the system of the confining radius $1.1 \sigma$ considered in Ref. [9]; one can observe an increase in the equilibrium density with pressure, and the existence of a breaking around $P^{*}$ $=14$ (instead of 15 for the $1.1 \sigma$ case $)$. The dashed line in the figure is the liquid branch of the Hall equation of state for a bulk system of hard spheres and was taken from Ref. [18]. The solid branch was not displayed because it starts at higher densities than the represented in the figure. It can be seen that for all the reduced pressures considered, the equilibrium densities are always lower than in bulk, an effect of the impossibility of the spheres to fill uniformly the space inside the tubes. All the confined arrangements considered in this paper have similar EOSs, with the main variations being the reduced pressures of the density breakings and the particular equilibrium densities at each pressure, both functions of the tube diameter. The discontinuities in the EOSs are associated with a change in the disposition of the spheres inside the tubes practically indistinguishable from a first-order transition. However, a careful study of the $1.1 \sigma$ case made in Ref. [9] suggests that it is not the case, favoring the conservative hypothesis of a crossover between two ordering patterns.

A first insight into what happens when pressure is raised is given in Fig. 2 for the same tube already considered in the previous figure. There, we display the radial density for three different pressures $\left(P^{*}=3\right.$ : full line; $P^{*}=8$ : dotted line; and $P^{*}=18$ : dashed line). We can see that even for the lowest pressure of the three, all the spheres are located in a shell whose maximum peak moves very little upon compression, with no obvious discontinuity associated with the abrupt change in density around $P^{*}=15$ displayed in Fig. 1 . The only difference is the width of the shell that narrows progressively, leaving a wider longitudinal hole in the center of the tube. This kind of hole has been observed experimentally in 


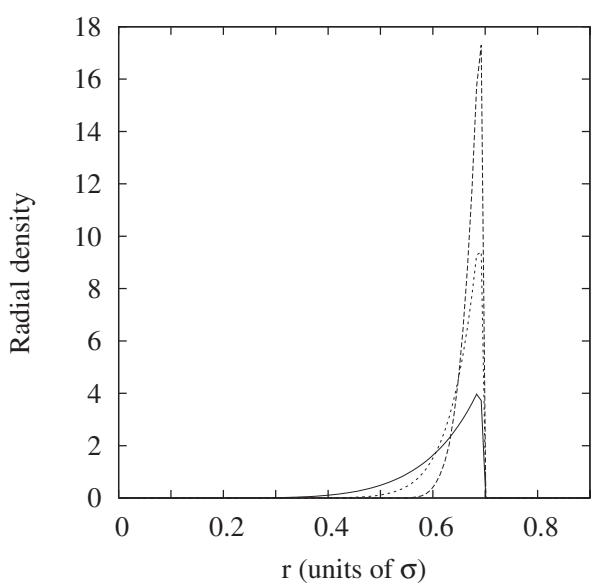

FIG. 2. Radial density (number of spheres per unit volume) for the same system than in the previous figure for $\beta P \sigma^{3}=P^{*}=3$ (solid line); $P^{*}=8$ (dotted line) and $P^{*}=20$ (dashed line). In all cases, we see only one sphere layer inside the tube. The volume of the cylinder is given in units of $\sigma^{3}$.

charge stabilized confined colloids [16]. Since the interaction between the particles and the wall is purely repulsive, the reasons of the formation of the layer are entropical; there is more space accessible to the spheres in the outer shell than in the smaller center of the cylinder axis, so it is more probable to find all sphere there.

To understand the nature of the change in the ordering, we will use the data in Table I and Fig. 3. In that table, we see the minimum radius needed to have $m$ hard spheres in the same plane. The limits have been obtained by simple geometrical arguments. In the figure we displayed the number of spheres per unit length, $m$, in units of $\sigma$ as a function of the reduced pressure. From top to bottom we have the case for $R=1.4,1.3,1.2$, and $1.1 \sigma$. The easiest one to interpret is the last one; when the pressure increases, the number of spheres in a slice of width $\sigma$ increases until its theoretical limit of three (see Table I) at $P^{*}=15$. The breaking at this pressure is due to the alternate rotation of the equilateral triangles formed to fit together in the closest way possible (see Fig. 4). If we suppose that the spheres in each of the triangles share the same plane and consider that the minimum distance between two spheres equals $\sigma$, a simple geometrical calculation gives us that the minimum distance between those planes is $0.8 \sigma$. That will produce an arrangement with and average of 3.75 spheres per $\sigma$ in the limit of perfect fitting versus the

TABLE I. Minimum radius to contain a set of $m$ coplanar spheres. A single number correspond to a ring of spheres located close to the tube wall. Two numbers indicate the number of spheres inside one of those rings (first number) and how many of them we can find inside that ring (number after the plus symbol).

\begin{tabular}{cc}
\hline \hline Confining radius $(\sigma)$ & Number of spheres $(m)$ \\
\hline 1.08 & 3 \\
1.21 & 4 \\
1.35 & 5 \\
1.50 & $6+1$ \\
\hline \hline
\end{tabular}

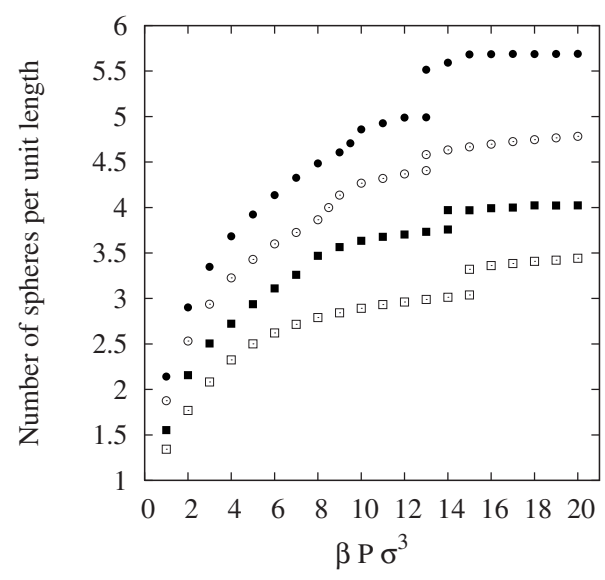

FIG. 3. Number of spheres per unit length (in units of $\sigma$ ) as a function of the tube radius in the same units. From top to bottom we have, $R=1.4,1.3,1.2$, and $1.1 \sigma$. The error bars are smaller than the size of the symbols and not shown for simplicity.

$\sim 3.5$ value at $P^{*}=20$ of Fig. 3. This difference could be due to the existence of defects that will destroy the long-range order, as found for this system in Ref. [9].

The case of $R=1.2 \sigma$ is different. According to Table I in this tube there is not enough room to have four coplanar spheres in the $x y$ plane. This means that the lower branch of the curve in Fig. 3 should end before that number. However, the upper part of it is basically a horizontal line for $m=4$. The reason could be deduced from Figs. 5 and 6. In Fig. 5 we displayed the normalized distribution of the cosine of the angle between two consecutive spheres and the center of the tube at $P^{*}=20$. The dotted lines are the simulation results and the dashed line is a fit to a Gaussian of mean -0.13 . The reason why the cosine distribution is not a delta is because what we have here are the real configurations including defects and allowing for some entropic disorder. This means that each sphere forms with its closest neighbor angles, which vary from $\sim 84^{\circ}$ to $\sim 114^{\circ}$, with a maximum probability at $\sim 98^{\circ}$. The normalized distribution of the $z_{n}$ distance between the same consecutive spheres is shown in Fig. 6 . There, the symbols and line have the same meaning as in the

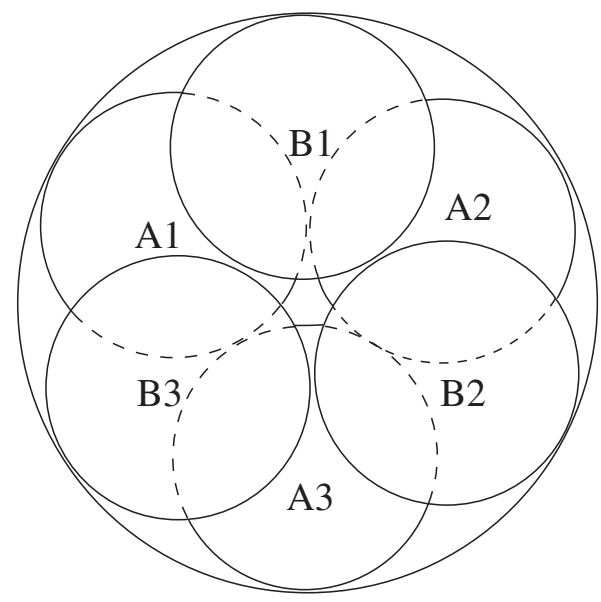

FIG. 4. Perfectly fitting triads of spheres in a maximum packet configuration for a cylinder of radius $R=1.1 \sigma$. 


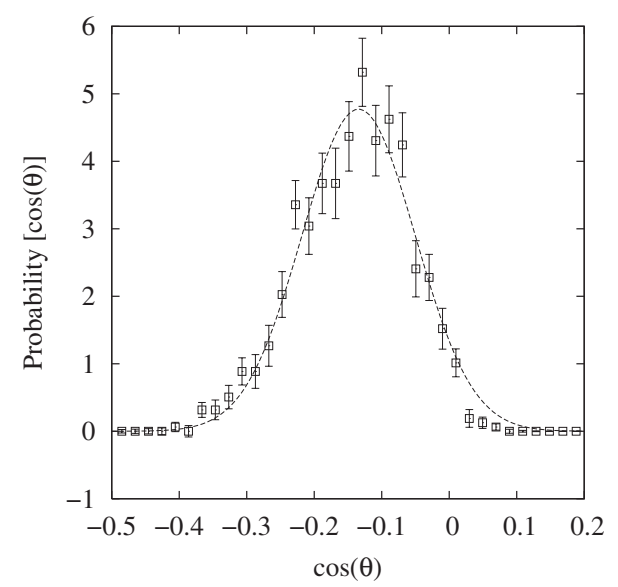

FIG. 5. Normalized distribution of the cosine of the angle formed by two consecutive spheres for a cylinder of $R=1.2 \sigma$ at $P^{*}=20$. Open squares: simulation results; dashed line: fit to a Gaussian. See further explanation in the text.

previous figure. The mean of the Gaussian is $0.24 \sigma$ within a range between 0.1 and $0.4 \sigma$. Both Figs. 5 and 6, together with the fact extracted from Fig. 2 that the spheres are located close to the wall, indicate the high regularity in the angles and distances typical of a helical distribution with some defects in the structure. The formation of this helix allows the spheres to explore the $z$ coordinate of every slab of length $\sigma$ and press as many of them as the corresponding to the next radius limit in Table I. A similar helix is formed in the high-pressure limit of the $R=1.3 \sigma$ tube. In Fig. 7 the full squares show the simulation results for this radius and $P^{*}$ $=16$. The fitting Gaussian now has a maximum around $0.21 \sigma$ and the distances in the $z$ axis are in the range $0-0.4 \sigma$. This indicates that with pressure, $m$ will increase until 5 , the number corresponding to the next limiting radius in Table I $(1.35 \sigma)$. The upper branch of the curve goes to this limit slower than in the $1.2 \sigma$ case, probably because the difference between the actual radius of cylinder and the closest radius in Table I is greater in the $1.3 \sigma$ tube. Since all helices are chiral structures, for those radii, those described above are chiral orderings.

This interpretation of how the spheres arrange with increasing pressure is fully compatible with the customary

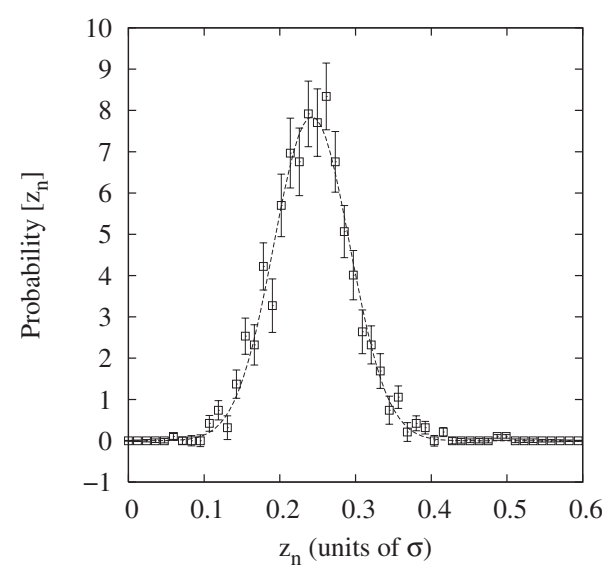

FIG. 6. Same as in the previous figure but for the distance in the $z$ axis between two consecutive spheres.

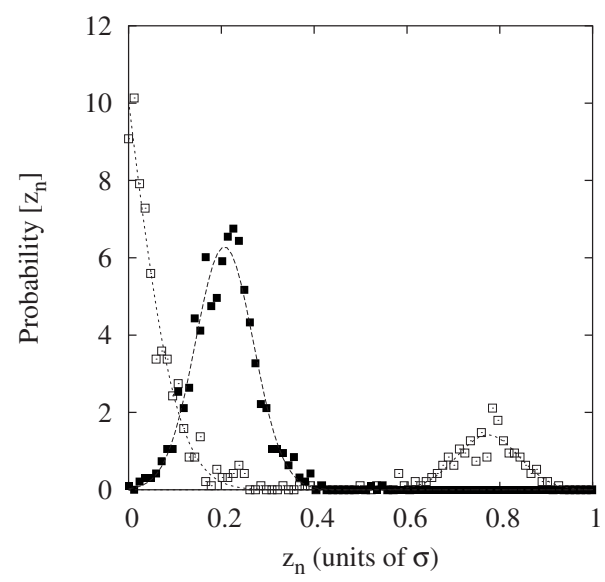

FIG. 7. Same as in Fig. 6 but for $R=1.3 \sigma$. Open squares: $P^{*}$ $=10$; full squares: $P^{*}=16$. Error bars are of similar size as in the previous figure and were not shown for simplicity.

view of a set of $m$ helices intertwined given in Refs. $[8,10,16]$, and obtained by visual inspection. Figures 8 and 9 (lower and upper parts, respectively) show representative snapshots corresponding to $P^{*}=20$ for $R=1.2$ and $1.3 \sigma$ that confirm the existence of such helices in our present case. Hodak and Girifalco [10] established that both schemes are algebraically compatible. This means that we can describe the high-pressure arrangements by means of a single helix, with given $a$ and $\theta$ parameters [see Eqs. (2) and (3)] common for all the $N$ spheres in the cylinder or, define $m$ subsets of $N / m$ spheres, each of them with the same $a^{\prime}$ and $\theta^{\prime}$ parameters, but different than the ones in the single helix case. The upper and middle parts of Fig. 8 give us an idea about the ordering process in the $R=1.2 \sigma$ cylinder; the loosely ordered structure at $P^{*}=6$ (upper part) orders itself in something similar to a columnar arrangement around $P^{*}=10$ (middle part) to be rearranged upon pressure as the helical structure already discussed at $P^{*}=20$ (lowest part).

The open squares in Fig. 7 give us the normalized distribution of $z$ distances for $R=1.3 \sigma$, but now for $P^{*}=10$. The pattern found does not correspond to a helix; there are two maxima, at $z \sim 0$ and $z \sim 0.77 \sigma$. This situation is similar for $R=1.2 \sigma$ in the pressure range between $P^{*}=10$ and the ordering breaking $\left(P^{*}=14\right.$ or $P^{*}=15$, depending on the tube). The only difference is in the location of the second maxima at the latter radius, at $0.46 \sigma$, and not shown for simplicity. The pattern in Fig. 7 suggests that in this pressure range, most of the spheres are located approximately on the same plane, with a minority of them trying to fit in the holes left. At

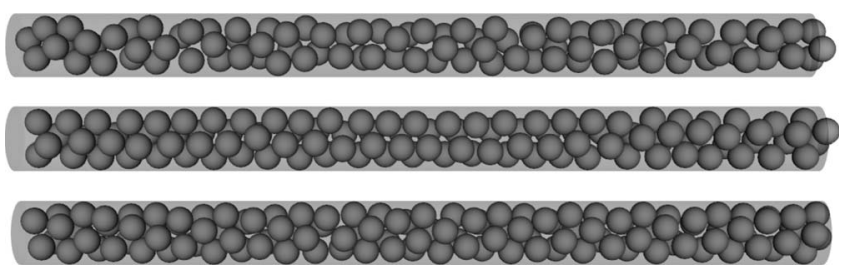

FIG. 8. Representative snapshots for some sphere arrangements inside a tube of $R=1.2 \sigma$ at different pressures. From top to bottom: $P^{*}=6,10$, and 20 . 


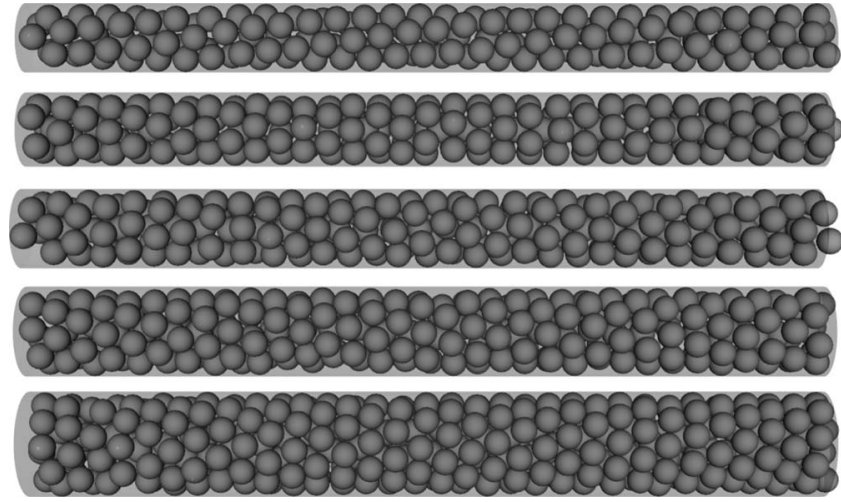

FIG. 9. Same as in the previous figure but for $P^{*}=20$ and different confining radii. From top to bottom: $R=1.3,1.4,1.5,1.7$, and $2 \sigma$.

lower pressures (below approximately $P^{*}=6$ ), the spheres form loose shells with $z_{n}$ distributions with wide Gaussians centered at $z=0$ (see the case for $R=1.4 \sigma$ below). In any of those structures, the angle between two consecutive spheres does not have a single peak as in the case of the helices discussed above.

\section{B. Wider tubes $(R>1.35 \sigma)$}

The sphere arrangement for the case of $R=1.4 \sigma$ and high pressures $\left(P^{*}>13\right)$ can be understood with the same tools as that in the previous cases; the pattern should approach that of the next limiting radius in Table $I$, in this case, a central sphere surrounded by five others $(R=1.5 \sigma)$. In fact, the big discontinuity around $P^{*}=13$ could be explained as the crossover between a single shell structure (considered in the previous section for thinner tubes), and one with some spheres forming a line in the center of the tube. This change can be visualized from the radial distribution functions of Fig. 10, in which we see the structures for $P^{*}=12$ and $P^{*}=20$. Given the fitting problems, in the center of the tube we only have a maximum linear density of $\sim 0.43 \sigma^{-1}$. Below $P^{*}=13$ and until $P^{*}=10$, we have a helical structure of the same kind of

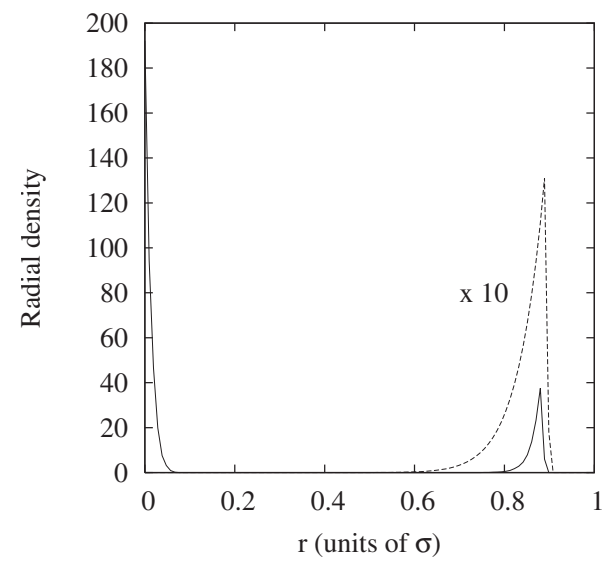

FIG. 10. Radial densities (number of spheres per unit volume in units of $\sigma^{3}$ ) for $R=1.4 \sigma$ for $P^{*}=20$ (full line) and $P^{*}=12$ (dashed line). This last case has been enlarged ten times for clarity.

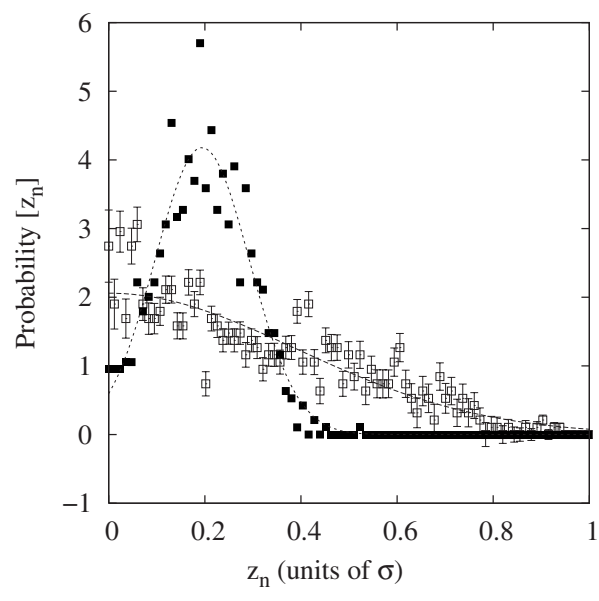

FIG. 11. Normalized distribution for the $z$ distance between consecutive spheres for $R=1.4 \sigma$ and $P^{*}=3$ (open squares) and $P^{*}=10$ (full squares). Dashed lines corresponds to fits to Gaussians centered at $z_{n}=0$ and $z_{n}=0.19$, respectively. In the last case, the error bars are similar to the ones for $P^{*}=3$ and not shown for simplicity.

the ones already seen for previous cases (full circles in Fig. $11, P^{*}=12$; maximum at $\sim 0.19 \sigma$, with an angle for the helix of $\sim 77^{\circ}$ ). The helical structure forms by compression of the structures at low pressures similar to the one depicted in Fig. 11 (open squares, $P^{*}=3$ ), in which the distributions are wide Gaussians centered at $z_{n}=0$, i.e., the spheres tend to accumulate in the same $z$ position with respect to each other. Between the loosely arranged structures and the helical one, we can find a crossover similar to that of the one we found for $R=1.3 \sigma$ at $P^{*}=8.5$. In both cases, changes are smoother than the corresponding density breakings found at $P^{*}>10$.

The rest of the figures are related to the cases with bigger radii than the ones considered until now. Figure 12 is similar to Fig. 3 but for (from top to bottom) $R=2,1.7$, and $1.5 \sigma$. As in Fig. 3, the error bars that are of the size of the symbols are not shown for simplicity. In all cases we observe a similar breaking in the number of spheres per unit length at different pressures $\left(P^{*}=11,12\right.$, and 13), which decreases with the radius increases. Below the breaking pressure we do not ob-

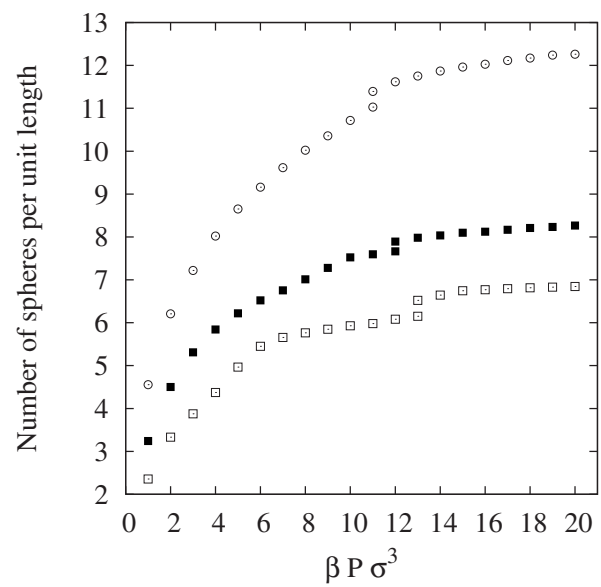

FIG. 12. Number of spheres per unit length (in units of $\sigma$ ) as a function of the tube radius. From top to bottom we have $R=2,1.7$, and $1.5 \sigma$ 


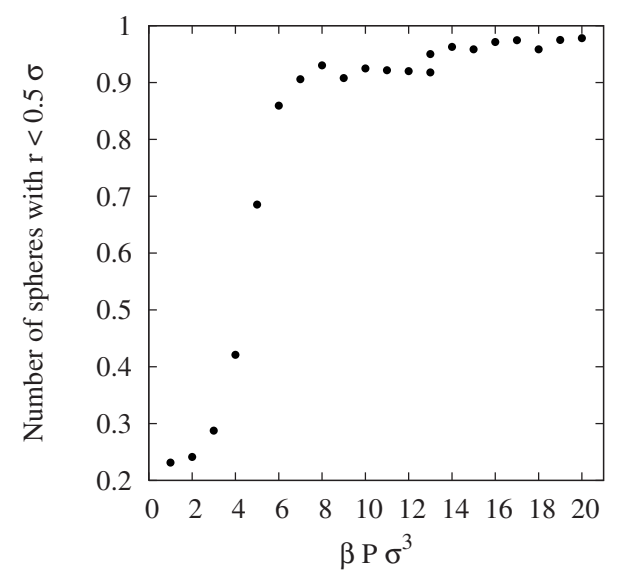

FIG. 13. Number of spheres per unit length located at a distance smaller than $0.5 \sigma$ from the center of a cylinder of $R=1.5 \sigma$. Error bars are of the size of the symbols.

serve any other discontinuity, but there is a noticeable change in the slope of the curve around $P^{*}=5$ for $R=1.5 \sigma$. The explanation to that is given in Fig. 13. There, we display the number of spheres for that radius located at a distance smaller than $0.5 \sigma$ from the center of the cylinder. The change in the slope starts at the same pressure at which the number of spheres per unit length in that location reaches a value close to its theoretical maximum of 1 . An inspection of Fig. 12 indicates that the high-pressure ordering pattern is such as to have seven spheres in the same slab or length $\sigma$, i.e., the maximum number allowed for this confining radius according to Table I. Since to have exactly seven spheres (one in the center and another six around the first one) we should have a perfect fit, and this is impossible, the spheres tend to form the $m=7$ intertwined helices indicated in the literature [10] and already mentioned for the cases $R=1.2$ and $1.3 \sigma$. However, a simple visual inspection of Fig. 9 suggests that at least the spheres located in the outer shell of the wider tubes $(R \geq 1.5 \sigma)$ can be thought of as located on a twodimensional triangular lattice wrapped around the different

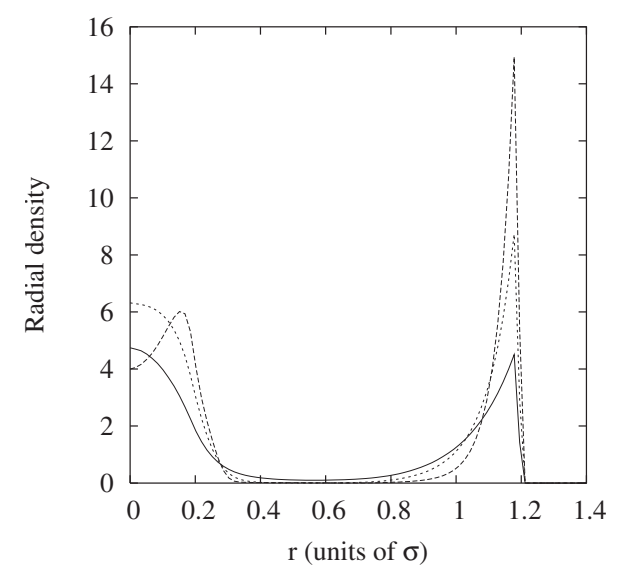

FIG. 14. Radial densities for the cases in which $R=1.7 \sigma$ for $P^{*}=3$ (solid line), $P^{*}=8$ (dotted line), and $P^{*}=20$ (dashed line). We can see that the separation between the outer layer and the central spheres could be safely established at around $0.6 \sigma$ for all pressures.

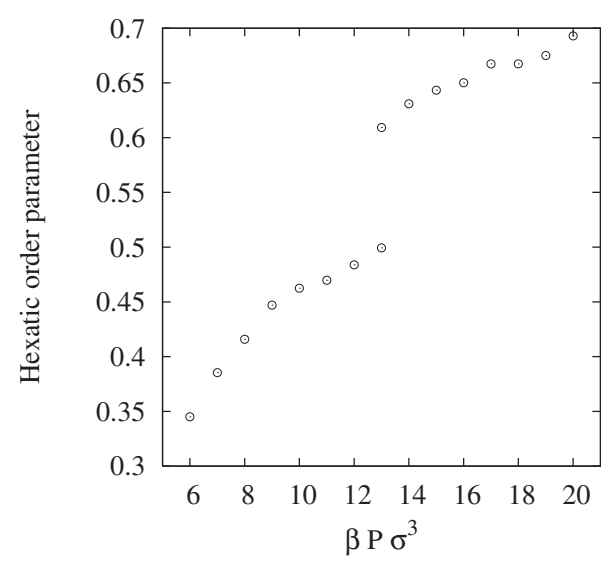

FIG. 15. Hexatic order parameter for the outer shell of spheres in a $R=1.5 \sigma$ tube. The breaking at $P^{*}=13$ coincides with the breaking in $m$ we can see in Fig. 12.

cylinders considered. To measure the quality of this approximation, we calculated the hexatic order parameter [Eq. (4)] for the spheres located in the outer layers of the cylinders of radii $R=1.5,1.7$, and $2 \sigma$. To determine which spheres were located on those shells, we resorted to radial distribution functions similar to the one depicted in Fig. 14 for $R=1.7 \sigma$. There, we can see that the limit between layers could be stated to be at $\sim 0.6 \sigma$ from the center of the tube for all pressures. The corresponding limits for $R=1.5$ and $2 \sigma$ are $\sim 0.5$ and $\sigma$, respectively.

The results for the hexatic order parameters are given in Fig. $15(R=1.5 \sigma)$ and Fig. $16(R=1.7$ and $2 \sigma)$. The error bars in both cases are of the size of the symbols and are not displayed for simplicity. In all cases we have an appreciable jump that coincides with the density breaking we observed in Fig. 12. This indicates that the change is at least partially due to the reordering of the structure of the outer layer upon compression to form quasi-two-dimensional sheets reminiscent of two-dimensional lattices on a curved surface. We should say, however, that the number of vacancies is considerable, since the value of this hexatic order parameter at the highest considered pressure is still pretty far for the value of one corresponding to a perfect triangular lattice.

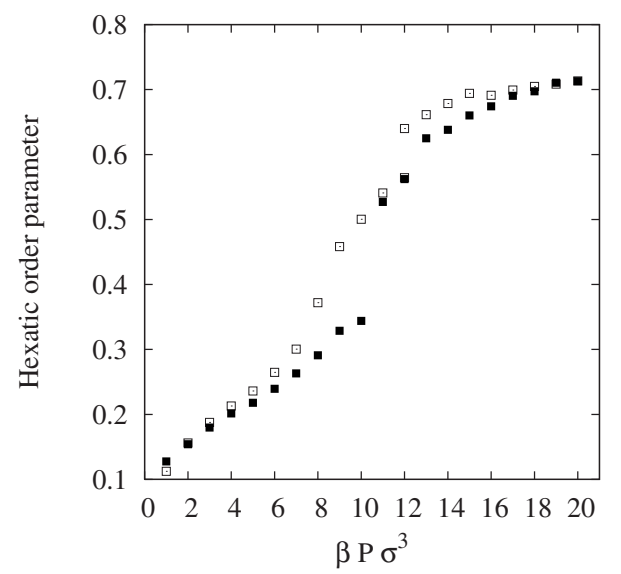

FIG. 16. Same as in the previous figure but for $R=1.7 \sigma$ (open squares) and $R=2 \sigma$ (full squares). 


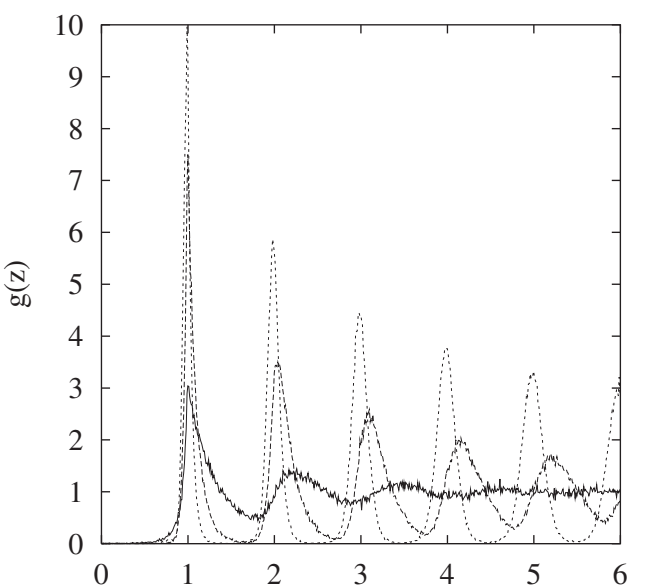

(a)

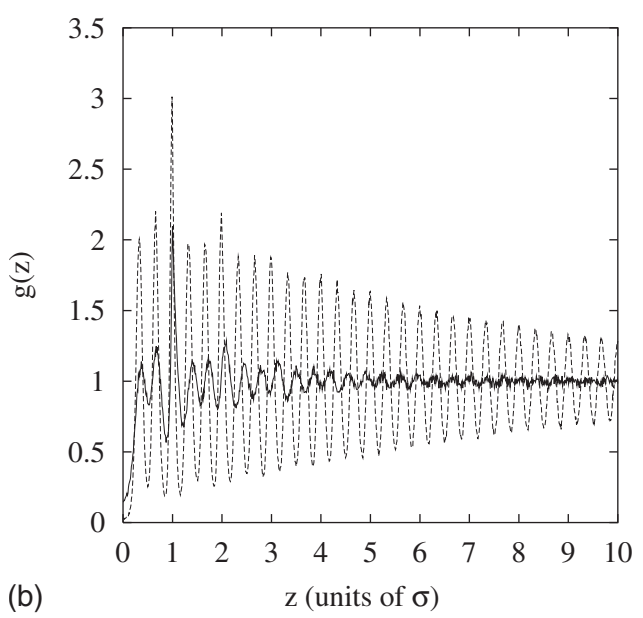

FIG. 17. Upper panel: radial distribution function along the $z$ coordinate for spheres located at a distance for the center lower than $0.6 \sigma$ for a tube or radius $1.7 \sigma$. Full line: $P^{*}=3$; dotted line: $P^{*}=8$; dashed line: $P^{*}=20$. Lower panel: same as $R=2 \sigma$ and a limiting inner radius of $\sigma$. Full line: $P^{*}=10$; dashed line: $P^{*}=20$.

To see if this ordering of the outer layer is the whole history, we should also consider what happens with the remaining spheres, i.e., the ones located inside the limiting radius used to define the outer shells. The $R=1.7 \sigma$ case is displayed in the upper panel of Fig. 17. There, we can see the change in behavior of the $g(z)$ functions for $P^{*}=3$ (full), 8 (dotted), and 20 (dashed line), but only for the spheres located inside an inner cylinder of radius $0.6 \sigma$. We do not see any discontinuity in the radial distribution function $(g(z))$ behavior with pressure, merely a compression, signaled by a decreasing in the distance between adjacent peaks. In this, those set of spheres behave similar to the ones confined in a smooth tube of $R<1.08 \sigma$, in which we do not have a density breaking when the pressure increases [9]. This means that the change in the ordering at $P^{*}=12$ for this radius is basically due to the ordering of the outer shell. This is exactly the opposite of what happens for the other case, $(R=2 \sigma)$. There, there is an appreciable change in the ordering of the inner spheres around the breaking density pressure $\left(P^{*}=11\right)$. In the lower panel of Fig. 17 we can see the nature of the change; upon compression the inner spheres order themselves to form a helical structure, indicated by the three peaks we can see for $z<\sigma$ in the $g(z)$ function at high pressures.

\section{CONCLUDING REMARKS}

We studied the ordering of hard spheres inside perfectly hard cylindrical pores. We found that for radii lower that 1.5 times the diameter of a hard sphere, the ordering pattern at high pressures is determined by the upper limit of how many spheres we can fit per unit length. This upper limit is given in Table I and was obtained by purely geometrical considerations. When the confining radius is such as to allow more than one cylindrical shell of particles, the high-pressure ordering depends on the formation of defected twodimensional triangular lattices wrapped to fit the corresponding tubes. In the case of $R=2 \sigma$, the symmetry breaking due to the reordering of the spheres is both due to the formation of this quasitriangular lattices and the ordering of the inner spheres to produce helical structures similar to those found inside smooth tubes of radius lower than $\sigma$ [8].

\section{ACKNOWLEDGMENTS}

The Spanish Ministry of Education and Science (MEC) and the Junta de Andalucia are thanked for financial support under Grant No. FIS2006-02356 and FEDER Funds, and group FQM-205, respectively.
[1] L. D. Gelb, K. E. Gubbins, R. Radshakrishnan, and M. Sliwinska-Bartkowiak, Rep. Prog. Phys. 62, 1573 (1999).

[2] H. Kanda, M. Miyahara, and K. Higashitani, Langmuir 16, 8529 (2000).

[3] R. Radhakrishnan, K. E. Gubbins, and M. SliwinskaBartkowiak, J. Chem. Phys. 116, 1147 (2002).

[4] J. Hoffmann and P. Nielaba, Phys. Rev. E 67, 036115 (2003).

[5] M. Rossi, D. E. Galli, and L. Reatto, Phys. Rev. B 72, 064516 (2005).

[6] F. R. Hung, B. Coasne, E. E. Santiso, K. E. Gubbins, F. R. Siperstein, and M. Sliwinska-Bartkowiak, J. Chem. Phys. 122, 144706 (2005).
[7] K. K. Mon and J. K. Percus, J. Chem. Phys. 112, 3457 (2000).

[8] G. T. Pickett, M. Gross, and H. Okuyama, Phys. Rev. Lett. 85, 3652 (2000).

[9] M. C. Gordillo, B. Martínez Haya, and J. M. Romero-Enrique, J. Chem. Phys. 125, 144702 (2006).

[10] M. Hodak and L. A. Girifalco, Phys. Rev. B 67, 075419 (2003).

[11] I. E. Kamenetskiy, K. K. Mon, and J. K. Percus, J. Chem. Phys. 121, 7355 (2004).

[12] K. Koga and H. Tanaka, J. Chem. Phys. 122, 104711 (2005).

[13] Y. Yin and Y. Xia, J. Am. Chem. Soc. 125, 2048 (2003). 
[14] S. A. Vanapalli, C. R. Iacovella, K. E. Sung, D. Mukhija, J. M. Millunchick, M. A. Burns, S. C. Glotzer, and M. J. Solomon, Langmuir 24, 3661 (2008).

[15] A. N. Khlobystov, D. A. Britz, A. Ardavan, and G. A. Briggs, Phys. Rev. Lett. 92, 245507 (2004).

[16] M. Tymczenko, L. F. Masal, T. Trifonov, I. Rodríguez, F.
Ramiro-Manzano, J. Pallarés, R. Alcubilla, and F. Meseguer, Adv. Mater. 20, 2315 (2008).

[17] R. Kitaura, N. Imazu, K. Kobayashi, and H. Shinohara, Nano Lett. 8, 693 (2008).

[18] D. Frenkel and B. Smit, Understanding Molecular Simulation (Academic, San Diego, 1996). 